\title{
Increasing User Attention with a Comic-based Policy
}

\author{
Madiha Tabassum, Abdulmajeed Alqhatani, Marran Aldossari, Heather Richter Lipford \\ University of North Carolina at Charlotte \\ Charlotte, North Carolina \\ [mtabassu, aalqhata, maldoss2, Heather.Lipford]@uncc.edu
}

\begin{abstract}
End user license agreements, terms of service agreements and privacy policies all suffer from many of the same problems: people rarely read them and yet still agree to whatever is contained within them. There are many usability challenges with these policies: they are often lengthy, with jargon filled language that is difficult to quickly comprehend. However, these notices are the primary tool for users to understand the privacy implications of their digital activities and make informed decisions on which websites and software they use. Prior research has explored alternative designs for such notices, using more visual and structured interfaces for conveying information. We expand upon these results by exploring a comic-based interface, examining whether it can engage users to pay more attention to a terms of service agreement. Our results indicate that the comic version did hold user attention for longer than text-based alternatives, encouraging deeper investigation into comic-based interfaces.
\end{abstract}

\section{ACM Classification Keywords}

H.5.2 Information Interfaces and Presentation: User InterfacesUser-centered design, Interaction styles; H.1.2 Models and Principles: User/Machine Systems-Human factors, Human information processing

\section{Author Keywords}

End User License Agreement, Terms of Service, Privacy policy, Privacy, Comic

\section{INTRODUCTION}

End User License Agreements (EULA) and Terms of Service agreements (ToS) are legal contracts between a purchaser or user of an application and the publisher or provider of that application. In addition to defining the terms and restrictions of that application, many agreements contain substantial information regarding how the provider will collect and use personal information, similar to a privacy policy. All of these notices are meant to educate users on data policies so that they can make informed decisions regarding whether and how they interact with different applications. However, there are well

Permission to make digital or hard copies of all or part of this work for personal or classroom use is granted without fee provided that copies are not made or distributed for profit or commercial advantage and that copies bear this notice and the full citation on the first page. Copyrights for components of this work owned by others than ACM must be honored. Abstracting with credit is permitted. To copy otherwise, or republish, to post on servers or to redistribute to lists, requires prior specific permission and/or a fee. Request permissions from permissions@acm.org.

CHI'18, April 21-26, 2018, Montreal, QC, Canada

(C) 2018 ACM. ISBN 978-1-4503-5620-6/18/04 .. \$15.00

DOI: https : //doi . org/10 . 1145/3173574 . 3173774 documented usability issues with all of these privacy-related notices. They can be difficult to comprehend because they are long, filled with technical language, and written at an advanced reading level $[1,2,3]$. As a result, users often make the reasonable choice that they are not worth the effort of reading, and mostly ignore them, even as they consent to them.

A key privacy protection mechanism is notice and choice, that is, consumers are to be made aware of the data policies of an organization in order to make choices over which of those policies they are willing to consent to, and thus which software and websites they use. End User License Agreements, Terms of Service agreements, and privacy policies are the primary methods for users to learn about those data policies in order to make that informed choice. The usability problems with existing privacy agreements and notices clearly limit consumers' ability to make such choices [4]. Indeed, various experiments have demonstrated that making privacy information more salient at the point of decision making can influence the decisions users make regarding which software to download or website to purchase from $[5,6]$. Thus, improving these agreements so that users are able to pay attention to and understand the information within them is an important goal for providing users with meaningful control over their privacy.

In this paper, we examine one proposal for engaging users to pay more attention to a privacy notice, using a comic to summarize the data policy. We hypothesize that the visual nature and story line of a comic may entice users to spend more time reading the agreement, and in turn lead to better understanding of that policy. We report the results of a labbased eye tracking study comparing the time and attention of users on a terms of service agreement. Our results indicate that there is an increase of attention, which we hope encourages additional investigation into whether this can lead to improved decision making.

\section{BACKGROUND}

The usability issues of EULAs and privacy policies have been well documented. Such policies are often lengthy [7], with jargon-filled language that can be hard to read and understand $[1,2,3,8]$. Actually reading such notices would take many minutes [9]. Thus, many users do not see sufficient benefit from paying attention to such notices [7,9] and subsequently spend very little time looking at them [10] or simply ignore them altogether [11]. Over time, users have also become habituated to these notices, further reducing user attention.

A number of researchers have proposed improvements for both EULA and privacy policy interfaces to improve user attention 
and understanding. For example, Kelley et al. proposed a Nutrition Label as a structured and visual representation and demonstrated that it increased users' accuracy in understanding a privacy policy $[12,13]$. Several studies have established that users perform better and prefer standardized and structured formats over the traditional long text notices [3, 13, 14]. Despite the benefits of these formats, many of these studies did not examine whether these alternative designs would sufficiently engage users to pay attention to them. With that goal in mind, Kay and Terry employed graphical design principles to draw user attention to important points within an End User License Agreement [15]. We examined this design within our study as well. Waddell also found that re-designing a EULA can improve user attitudes, which correlates with comprehension [14]. Other researchers have proposed methods to draw user attention to important text by using visual attractors [16, 17], as well as forcing users to interact with [16, 17] or look at [18] key pieces of text. However, these technique would only work for small amounts of text, not an entire policy.

Comics have previously been used in interfaces to engage users to learn about different topics, such as how not to fall for a phishing attack [19], Internet security risks [20], recommended online privacy behaviors [21], and patient care education [22]. Zhang-Kennedy et al. demonstrated that interactive comics can increase user comprehension of security-based education, and lead to behavior change [23]. Knijnenburg and Cherry proposed to use comics as a more inviting, understandable and engaging medium to improve communication of privacy notices [24]. However, they did not report any evaluations. In our proposed interface, we use a comic as a summary of the most important Terms of Service. Designing a visual interface with little text will be unlikely to ever contain all of the lowlevel details in a full ToS. However, previous works [5, 7, 12] indicate that shortened or structured forms of policies may better support decision making. Thus, our goal in this study is to investigate whether a comic could engage users and lead to more attention and comprehension of a privacy notice.

\section{METHODOLOGY}

Our study aims to examine the potential of a comic-based Terms of Service agreement in holding user attention longer than plain text, which in turn may lead to better comprehension of the agreement. In addition to the comic and text agreement, we compared another visual alternative, the textured agreement [15]. Similar to other such studies, we designed our study to use deception so the ToS was not the user's primary task $[15,14]$, yet with the addition of an eye-tracker to more fully examine the user's focus of attention. We have used a standard Tobii eye tracker for our study.

Using an eye-tracker required us to make several study design decisions. First, we needed to conduct the study in a lab setting. We also decided to limit the agreement to one page, so there would be no scrolling. Thus, rather than a full agreement, all three conditions utilized a summary of a full agreement (with a link available for participants to view an entire text agreement). Finally, we simulated registration at a website that gathered a small amount of personal information even as

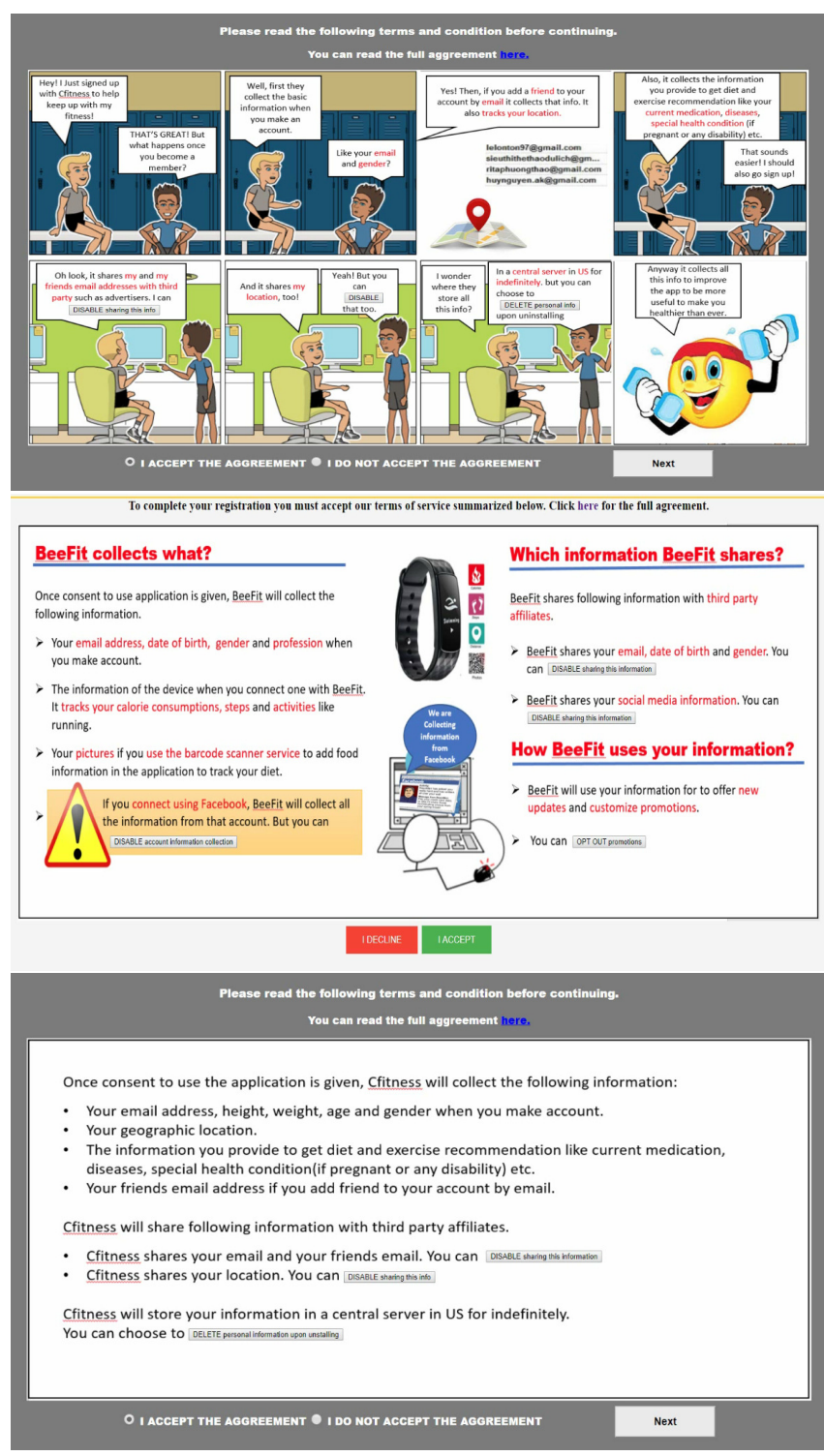

Figure 1. Comic, Textured and Text based ToS summary interface

part of creating an account, so participants would think that their information was potentially being used.

Figure 1 shows an example of each condition. We created two fake health and fitness websites (each with different names and designs), with registration pages that asked for name, email, and two other pieces of personal information. The ToS summary page was then displayed in one of three formats text, textured, or comic - with a link to a full text agreement. The comic was developed in Pixton ${ }^{1}$. We created a different policy for each website regarding what information the application will collect and why; what information it will share and with whom; and how it's storing the collected information. We aimed for similar complexity and policy length for each website, and also sought to put the same information in each format. While the comic has extensive graphics which may

\footnotetext{
${ }^{1}$ https://www.pixton.com/
} 
attract a participant's attention, it still contains a significant amount of text, and thus did not take as much advantage of graphical representations or a story-line as a comic could. The text-only summary used bulleted lists, and the textured ToS was a middle-ground, still very structured but with more color and graphics. All formats contained buttons to opt out of certain data collection and use.

Participants were recruited in person, as they entered one academic building, the library, or student union on our campus and asked to take part in a study examining how people perceive and use recommendations from health and fitness applications. Participants were asked to verbally consent to participating and being recorded. To try to increase the ecological validity of the study, participants were told that after registering for accounts on two websites, they would have the opportunity to install those applications on their own mobile devices. They were also informed that study personnel were not responsible for any information they provided to the applications. They were told that we would use an eye tracker to record their interactions with the websites. They were then asked to to register for accounts on each of the two websites, where the ToS summary was then displayed as a part of that task. Upon completing registration at the first website, the site displayed that an email would be sent to complete the registration process. The study investigator then asked the participant to register at the other website while waiting for that email. Thus, participants saw two different conditions. We counterbalanced the order of conditions, and assigned participants round robin to each of the six possible condition pairings, attempting to ensure equal numbers for each pairing.

After completing the second registration, participants were then debriefed on the real purpose of the study and given a written consent agreement, or the option of leaving the study. Finally, participants completed a survey with both a comprehension test for each website's terms of service agreement, and perceptions of each of the two formats they saw. The study was completed within 20 minutes, and participants were compensated with a $\$ 5$ gift card for a coffee chain. The study was approved by the university IRB.

\section{Performance Measures}

We gathered three different metrics for examine performance:

- Exposure time: On the website, we logged the amount of time participants spent on the ToS summary page.

- Attention: We collected eye gaze data, specifically the number of fixations on each agreement page.

- Comprehension: We gave participants a quiz of 4 questions per website on the content of the ToS agreement.

\section{RESULTS}

60 participants were recruited from the University of North Carolina at Charlotte campus. With two conditions per participant, we had 39 subjects who interacted with the comic, 39 with the textured summary agreements and 42 with the plain text summary. 37 of the participants were male, and 23 were female. $91.7 \%$ of the participants were aged $18-27$ years old, and the remainder were $28-47$ years old. The sample was primarily students $(88.33 \%)$.

As each participant contributed data to two conditions, observations are not independent. So we fitted our data to a linear mixed model to account for the repeated measures. We used a Bonferroni correction for all post-hoc comparisons.

\begin{tabular}{|c|c|c|c|}
\hline Measure & Comic & Textured & Text \\
\hline Time on ToS & 37.31 & 25.28 & 21.62 \\
(in seconds) & SD: 23.78 & SD:17.78 & SD: 15.82 \\
\hline Eye gaze fixation & 30.42 & 19.35 & 17.56 \\
count & SD:17.88 & SD:14.74 & SD:14.43 \\
\hline Quiz score & 2.23 & 2.05 & 2.14 \\
(out of 4) & SD:1.26 & SD:1.05 & SD:1.02 \\
\hline
\end{tabular}

Table 1. Summary of different measures across conditions

\section{Exposure Time}

Table 1 summarizes the mean time users spent on each agreement format. The comic condition shows the longest time, followed by the textured condition. For all conditions, there was a large standard deviation, with some participants spending only a few second, and some spending more than a minute. A linear mixed model analysis with agreement condition as the fixed factor and time on the page as the dependent variable showed a significant difference between conditions, $\mathrm{F}(2,58)=8.817$, $\mathrm{p}=.001$. The post hoc comparisons indicate significant differences between the comic and textured $(\mathrm{p}=.028)$, and comic and plain text $(\mathrm{p}=.001)$ conditions. There were no statistically significant differences between the textured and text summaries $(\mathrm{p}=0.933)$.

\section{Attention}

For our analysis on user attention, we relied on measures related to gaze fixation with a minimum threshold of $100 \mathrm{~ms}$. The eye tracker did not function properly at all for 7 participants and in one condition for 2 participants. Because a linear mixed model uses maximum likelihood estimation, we included the participants with data for only one condition in the analysis. We found one outlier (more than two standard deviations away) for the text condition when compared against both the within-condition and text-condition mean. We excluded the data for that participant from our analysis.

The average number of fixations per participant is reported in Table 1. We did not include the participants with the missing values (3.7\% of the sample) in calculating the mean fixation count. The analysis with the mixed linear model with agreement condition as the fixed factor and eye gaze fixation count as the dependent variable was significant, $F(2,50)=12.926$, $\mathrm{P}=0.001$. Post hoc comparisons indicate significant differences between the comic and textured $(\mathrm{p}=.001)$ and comic and plain text $(\mathrm{p}<0.001)$ conditions. Again, there was no statistically significant difference between textured and text summary conditions.

In addition to the quantitative metrics, we generated an aggregated heatmap of eye gaze fixations on various regions of each of the agreements using iMotion software as shown in Figure 2 . The red regions of the map display the areas participants 

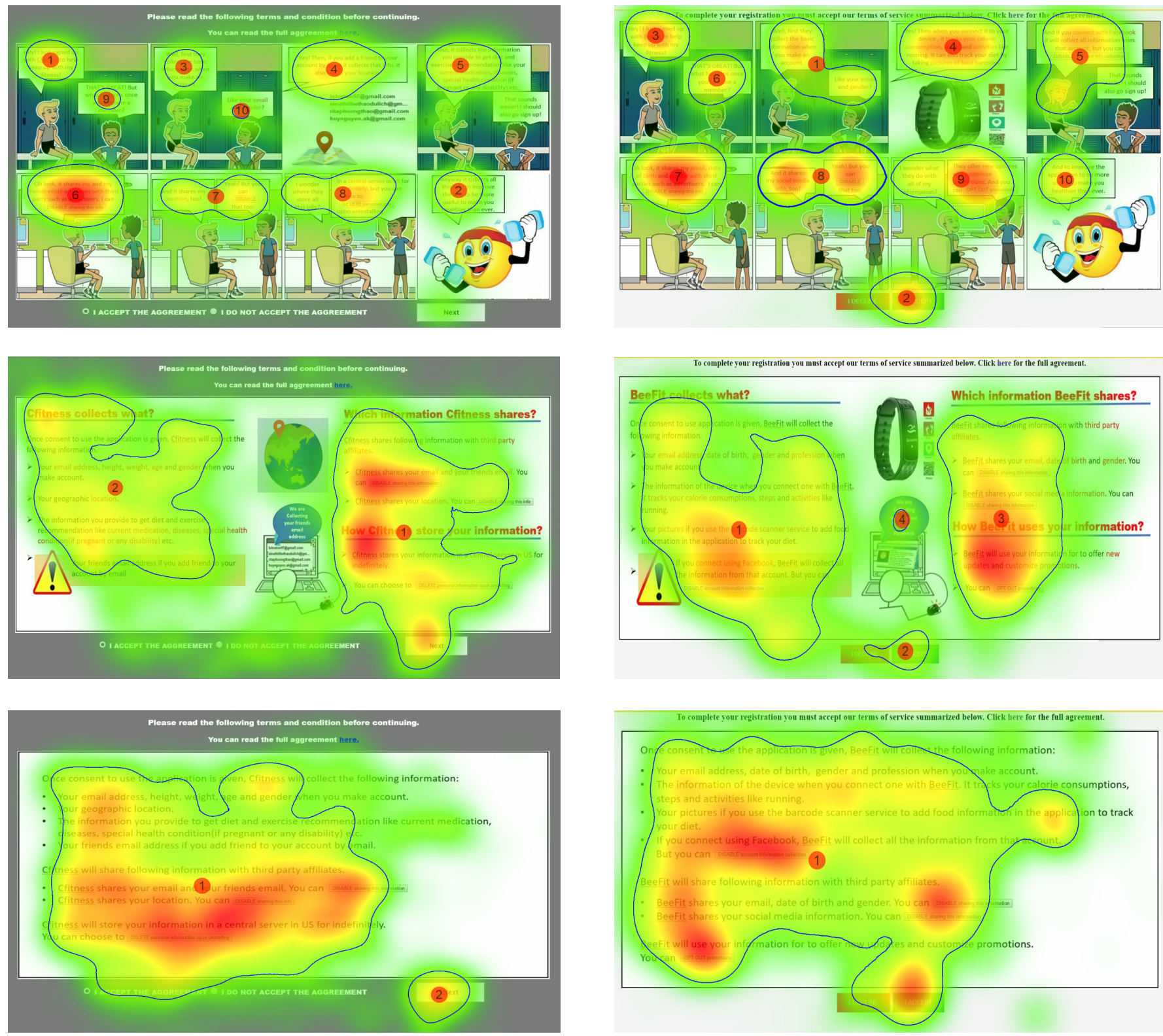

Figure 2. Aggregated heatmaps of eye gaze fixations. Row 1: Comic ToS (Interface 1: 18 participants, Interface 2: 16 participants). Row 2: Textured ToS (Interface 1: 18 participants, Interface 2: 17 participants). Row 3: Textured ToS (Interface 1: 17 participants, Interface 2: 20 participants)

looked for longer while the green regions are where participants looked for shorter durations. The eye tracking software also computes areas of interest, shown in the black lines in Figure 2. In the text and textured agreements, participants focused most on the left and bottom of the page, while in the comic agreements participants focus was scattered throughout the agreement on the text. In examining individual heatmaps, we observed that users frequently looked at all or most of the text bubbles in the comic condition, but rarely at all of the text in either of the two other conditions.

Only three participants went to the full terms of services page. Participants did pay attention to the disable buttons. For the text and textured agreements, there is higher attention inten- sity in the locations of the disable buttons. Out of 60 participants, 25 clicked on at least one disable button across the two websites. 14 participants clicked disable buttons for both agreements. Of the rest: 5 participants were from the comic condition, three from the textured condition and three from the text condition.

\section{ToS Comprehension}

Participants' scores on the comprehension quiz are also reported in Table 1. As can be seen in the table, we found no differences between the interface formats regarding ToS comprehension. However, using a Pearson correlation, we did find that participants' scores are significantly correlated 
with the amount of time they spent on the ToS agreement page $(\mathrm{r}=.324, \mathrm{p}<0.001)$. This echoes the findings of prior work, that more time spent on an agreement can lead to improved understanding [15]. Yet, in this study, the correlation was not large enough to cause a difference in participants' test scores among the different ToS formats. The short quiz may have also not allowed for sufficient differentiation between participants.

\section{Qualitative Feedback}

In the questionnaire, participants were asked which ToS interface they liked most between the two they saw. Of the 39 participants who saw the comic version, 22 preferred it. 14 preferred the textured agreement, and another 14 preferred the text format. The remainder could not decide between the interfaces.

A number of participants $(n=12)$ reported that the comic format was visually attractive or more engaging. One commented:

"It made what otherwise would be a dull read an entertaining setting that allowed me to understand what the terms were about."

However, several participants $(n=3)$ did not find the comic format appealing as suggested by a few comments:

"A lot of information cluttered on one screen which made it hard for me to follow along with."

\section{LIMITATIONS}

Our study was conducted in a laboratory setting, and thus has a number of limitations. First, the sample was mostly young adults who may be more technologically savvy than the general population. Moreover, almost all the participants were students. Second, although we told participants we were not responsible for the information they shared, the perceived threat is likely still relatively low. Because of the artificial nature of the study setting, we suspect that the participants would assume the websites had been vetted and that their information would be safe. Thirdly, the sample size is still small although on par with other similar studies. Finally, we utilized a summary of a ToS instead of the full ToS agreement. While users may be more likely to pay attention to a shorter summary than a lengthy agreement, a summary may not provide all information that is relevant to the user.

\section{DISCUSSION AND CONCLUSION}

In general, our study was successful. Participants spent longer and paid more attention to the comic based ToS agreement than the textured and plain text agreements. Still, the gains are modest, as participants only spent around 10 additional seconds reading the agreement, with approximately 10 more eye gaze fixations. The comic format was also preferred by more participants, but others instead found the format overwhelming. However, we have no evidence of the end goal improving user understanding. User comprehension did not improve, although the questionnaire used for this measure may have been too short to reveal differences.

We were surprised that the text and textured ToS agreements performed similarly on all measures. The addition of color and graphics on the textured format did not appear to draw additional attention, and did not appear to impact what users focused on for the most part. Unlike the previous evaluation of the textured agreement [15], our text comparison was already short and structured. Thus, our results imply that a short and structured format may be what is most important for users. As one participant stated:

\section{"(I liked the plain text ToS because) the information was pre-} sented clearly and in an organized structure."

This echoes the findings of prior work that shorter text is preferred by users [14].

A comic ToS agreement is new to users, and thus novelty may have contributed to our results. However, we also found that many participants spent longer than we expected on the text format, and did not completely ignore it. All formats in our study were somewhat novel, users do not normally see even the short, bulleted text list we displayed. Thus, novelty and the study setting may have led to participants spending more time than usual on the ToS agreement, and time spent was correlated to comprehension. Our results suggest possible benefits from a comic, yet these should be validated with more real world tasks. In addition, this study did not examine habituation. Users may eventually ignore even shorter and more engaging formats if they do not perceive the benefits of such information. However, we believe the comic-based formats have more potential for variations, hence tackling habituation better than textured and text-based formats. Yet designing an engaging and novel comic requires additional design effort. Determining the guidelines for counteracting habituation within a comic is another important research question for future work.

Our results so far imply that taking the approach of displaying important information to a user using a more graphical design rather than lengthy jargon can improve attention and the average time spent reading ToS agreements. This initial comic design was very basic, with lots of text. While not unrealistic, it does not take full advantage of the affordances of comics to engage and communicate to users. We believe simpler and more graphical comics with reduced text will further benefit users. However, organizations would need guidelines and assistance for creating such summaries. They may also need incentives to do so. Future research will need to investigate what motivates the service providers to provide more usable policies to their users, and guidelines that are useful and actionable for them to create such interfaces. Finally, our ultimate goal is to help users make more informed decisions. As yet, we have found no evidence that user understanding is improved with the comic and we did not examine actual decision making in our study. We hope this study provides additional evidence that novel privacy notice designs warrant additional investigation, and encourage future studies to examine how to support privacy decision making.

\section{ACKNOWLEDGMENTS}

This work was partially supported by NSF award \#1461166. We thank Jonathan Jeremie and Tommy King for their work in creating the interfaces and piloting the study. 


\section{REFERENCES}

1. Ewa Luger, Stuart Moran, and Tom Rodden. Consent for all: Revealing the hidden complexity of terms and conditions. In Proceedings of the SIGCHI Conference on Human Factors in Computing Systems, CHI '13, pages 2687-2696. ACM, 2013.

2. Carlos Jensen and Colin Potts. Privacy policies as decision-making tools: An evaluation of online privacy notices. In Proceedings of the SIGCHI Conference on Human Factors in Computing Systems, CHI '04, pages 471-478. ACM, 2004.

3. Aleecia M. Mcdonald, Robert W. Reeder, Patrick Gage Kelley, and Lorrie Faith Cranor. A comparative study of online privacy policies and formats. In Proceedings of the 9th International Symposium on Privacy Enhancing Technologies, PETS '09, pages 37-55. Springer-Verlag, 2009.

4. L. F. Cranor. Necessary but not sufficient: Standardized mechanisms for privacy notice and choice. Journal of Telecommunications and High Technology Law, 10(2), 2012.

5. Nathaniel S. Good, Jens Grossklags, Deirdre K. Mulligan, and Joseph A. Konstan. Noticing notice: A large-scale experiment on the timing of software license agreements. In in Proceedings of the ACM Conference on Human Factors in Computing Systems (CHI 2007. ACM Press, 2007.

6. Serge Egelman, Janice Tsai, Lorrie Faith Cranor, and Alessandro Acquisti. Timing is everything?: The effects of timing and placement of online privacy indicators. In Proceedings of the SIGCHI Conference on Human Factors in Computing Systems, CHI '09, pages 319-328. ACM, 2009.

7. Nathaniel Good, Rachna Dhamija, Jens Grossklags, David Thaw, Steven Aronowitz, Deirdre Mulligan, and Joseph Konstan. Stopping spyware at the gate: A user study of privacy, notice and spyware. In Proceedings of the 2005 Symposium on Usable Privacy and Security, SOUPS '05, pages 43-52. ACM, 2005.

8. L. F. Cranor. P3p: making privacy policies more useful. IEEE Security Privacy, 1(6):50-55, Nov 2003.

9. A.M. McDonald and L.F. Cranor. The cost of reading privacy policies. 4:540-565, 012008.

10. Thomas Maronick. Do consumers read terms of service agreements when installing software?a two-study empirical analysis. International Journal of Business and Social Research, 4(6), 2014.

11. Z. Kaupas and J. Ceponis. End-user license agreement-threat to information security: a real life experiment. In Proceedings of the IVUS International Conference on Information Technology, pages 55-60, 2017.

12. Patrick Gage Kelley, Joanna Bresee, Lorrie Faith Cranor, and Robert W. Reeder. A "nutrition label" for privacy. In Proceedings of the 5th Symposium on Usable Privacy and Security, SOUPS '09, pages 4:1-4:12. ACM, 2009.
13. Patrick Gage Kelley, Lucian Cesca, Joanna Bresee, and Lorrie Faith Cranor. Standardizing privacy notices: An online study of the nutrition label approach. In Proceedings of the SIGCHI Conference on Human Factors in Computing Systems, CHI '10, pages 1573-1582. ACM, 2010.

14. T. Franklin Waddell, Joshua R. Auriemma, and S. Shyam Sundar. Make it simple, or force users to read?: Paraphrased design improves comprehension of end user license agreements. In Proceedings of the $2016 \mathrm{CHI}$ Conference on Human Factors in Computing Systems, CHI'16, pages 5252-5256. ACM, 2016.

15. Matthew Kay and Michael Terry. Textured agreements: Re-envisioning electronic consent. In Proceedings of the Sixth Symposium on Usable Privacy and Security, SOUPS '10, pages 13:1-13:13. ACM, 2010.

16. Cristian Bravo-Lillo, Lorrie Cranor, Saranga Komanduri, Stuart Schechter, and Manya Sleeper. Harder to ignore? revisiting pop-up fatigue and approaches to prevent it. In 10th Symposium On Usable Privacy and Security (SOUPS 2014), pages 105-111, Menlo Park, CA, 2014. USENIX Association.

17. Cristian Bravo-Lillo, Saranga Komanduri, Lorrie Faith Cranor, Robert W. Reeder, Manya Sleeper, Julie Downs, and Stuart Schechter. Your attention please: Designing security-decision uis to make genuine risks harder to ignore. In Proceedings of the Ninth Symposium on Usable Privacy and Security, SOUPS '13, pages 6:1-6:12, New York, NY, USA, 2013. ACM.

18. Yousra Javed and Mohamed Shehab. Look before you authorize: Using eye-tracking to enforce user attention towards application permissions. 2017, 042017.

19. Ponnurangam Kumaraguru, Steve Sheng, Alessandro Acquisti, Lorrie Faith Cranor, and Jason Hong. Teaching johnny not to fall for phish. ACM Trans. Internet Technol., 10(2):7:1-7:31, June 2010.

20. Sukamol Srikwan and Markus Jakobsson. Using cartoons to teach internet security. Cryptologia, 32(2):137-154, April 2008.

21. Leah Zhang-Kennedy, Khadija Baig, and Sonia Chiasson. Engaging children about online privacy through storytelling in an interactive comic. 2017.

22. Michael Green and Kimberly R Myers. Graphic medicine: Use of comics in medical education and patient care. 340, 032010.

23. Leah Zhang-Kennedy, Sonia Chiasson, and Robert Biddle. The role of instructional design in persuasion: A comics approach for improving cybersecurity. International Journal of HumanâĂSComputer Interaction, 32(3):215-257, 2016.

24. Bart P. Knijnenburg and David Cherry. Comics as a medium for privacy notices. In WSF@SOUPS, 2016. 